\title{
EXAMINING DIFFERENTIAL ITEM FUNCTIONING DUE TO ITEM DIFFICULTY AND ALTERNATIVE ATTRACTIVENESS
}

\author{
Paul Westers and Henk Kelderman \\ UNIVERSITY OF TWENTE
}

\begin{abstract}
A method for analyzing test item responses is proposed to examine differential item functioning (DIF) in multiple-choice items through a combination of the usual notion of DIF, for correct/incorrect responses and information about DIF contained in each of the alternatives. The proposed method uses incomplete latent class models to examine whether DIF is caused by the attractiveness of the alternatives, difficulty of the item, or both. DIF with respect to either known or unknown subgroups can be tested by a likelihood ratio test that is asymptotically distributed as a chi-square random variable.
\end{abstract}

Key words: differential item functioning, multiple choice items, Rasch model, guessing model, incomplete latent class model, goodness-of-fit testing.

\section{Introduction}

Items in educational or psychological tests may show differential item functioning (DIF). This means that the probability of a correct response among equally able test takers is different for various racial, ethnic, or gender subgroups. A given educational or psychological test consisting of many items with significant DIF may be unfair for certain subgroups, and it is important to identify these items, so that they can be improved or deleted from the test. Many DIF detection methods have been proposed since Binet and Simon (1916) drew attention to this problem. Reviews of previous DIF (also called item bias) detection methods are given by Rudner, Getson, and Knight (1980), Berk (1982), and Osterlind (1983).

In the last decade, the DIF detection methods have been improved to provide a better basis for matching on ability. Various methods have used the number correct score of the test for this ability matching (Holland \& Thayer, 1986; Mellenbergh, 1982; Scheuneman, 1979). Recently, DIF detection methods have been proposed that are based on item response theory (IRT) (Baker, 1977; Lord, 1980; Muthén \& Lehman, 1985; Wright, Mead, \& Draba, 1975). Thissen, Steinberg and Wainer (in press) give an overview of IRT-based DIF detection methods and demonstrate their use. They also discuss DIF detection methods that can be used with multiple choice items, where the response alternatives are also potential sources of DIF.

Green, Crone, and Folk (1989) focus on the differential attractiveness of the incorrect responses (or "distractors"). If a particular distractor is more attractive to subjects from one subgroup than from another, Green et al. conjecture that "... the item probably means something different to the different groups"' (p. 147). They perform a loglinear analysis of the subgroup $\times$ score group $\times$ incorrect response contingency table for each item, to detect distractors that are more popular in one subgroup than in another. A similar approach of Veale and Foreman (1983) is based on the notion that examinees' responses to the incorrect alternatives provide more and better information concerning DIF than their responses to the correct alternative. Their model, 
called the overpull probability model, incorporates parameters representing (a) achievement differences across groups and (b) differences in alternative difficulty. Their proposed method also indicates the likely source of the bias so that the item may be revised to eliminate the bias rather than discarding the item. The methods proposed by Green et al. and Veale and Foreman have certain drawbacks; for example, the Green et al. method is not based on an IRT model and the Veale and Foreman method does not control for ability. The DIF detection method proposed in this paper will avoid these two problems.

Another source of DIF in multiple choice items is concerned with the differential difficulty of the problem to be solved. An item may show DIF if it is more difficult for some subgroup than for others, even if they are equally able on the trait of interest (Lord, 1980; Rudner, Getson, \& Knight, 1980). In this paper, a DIF detection method is described that separates both sources of bias. In the proposed method, a distinction is made between a "Know" state in which the subject has complete knowledge of the answer and a "Don't know" state. Furthermore, it is assumed that if the subject is in the "Know" state, the subject will give the correct answer. Here the probability that the subject is in the "Know" state is assumed to be governed by the Rasch (1960) model. If the subject is in the "Don't Know" state, the subject will guess the most attractive alternative, where the attraction of an alternative may be different for different alternatives, including the correct one.

The proposed DIF detection method differs from that of Thissen, Steinberg, and Fitzpatrick (1989), who distinguish between a "Don't know" state and a state in which the subject has partial knowledge or complete knowledge of the answer. In the "Don't know" state, the subject guesses the answer as before, but in the "Partial knowledge" state, the subject may select a response alternative according to response probabilities that are governed by Bock's (1972) nominal response model.

The proposed method is simpler than that of Thissen, Steinberg, and Fitzpatrick (1989). This simplicity has two advantages. First, it contains fewer parameters; for example, for a four-choice item, the proposed model has five item parameters while the model of Thissen et al. has fourteen. Obviously, if the sample is not very large, the parameters of the model by Thissen et al. cannot be estimated reliably. So, in that case, one may be inclined to "buy information by assumption" and use the simpler model. Second, the proposed model can be easily formulated as a latent class analysis (LCA) model (Kelderman, 1988). LCA models have been used extensively for measurement in sociology, psychology, and education (Clogg, 1981). There is a well-developed theory for maximum-likelihood estimation and likelihood-ratio testing of the LCA models (Goodman, 1978; Haberman, 1979; Lazarsfeld \& Henry, 1968). By comparing the fit of different LCA models, DIF in the attraction of the alternatives and DIF in the parameters of the Rasch model can be tested separately (Kelderman, 1989; Kelderman \& Macready, 1990). Also, the model can be extended to latent classes, so that the subgroups for which an item exhibits DIF may be latent.

In what follows, a model for multiple choice items is developed and formulated within the latent class framework. Different models for the detection of DIF are formulated, including a provision for defining the subgroup as a latent variable. A computationally efficient estimation method is described and illustrated with empirical data.

A Model for Multiple Choice Items that Accounts for Selection of Each Alternative

Suppose that each subject, randomly drawn from a population of subjects, responds to $k$ test items, where the answer to item $j$ may be any one of the $r_{j}$ responses, 
denoted by $y_{j}\left(y_{j}=1, \ldots, r_{j}\right)$. Let $x_{j}$ indicate the latent response of the subject, taking values $x_{j}=1$ if the subject is in the "Know" state (i.e., the subject has complete knowledge of the answer), or $x_{j}=0$ if the subject is in the "Don't know" state. The random variables associated with $y_{j}$ and $x_{j}$ are denoted by $Y_{j}$ and $X_{j}(j=1, \ldots, k)$, respectively.

The relationship between the latent response $x_{j}$ and the observed response $y_{j}$ is described by the conditional probability

$$
\Phi_{x_{j} y_{j}}^{X_{j} Y_{j}} \equiv P\left(y_{j} \mid x_{j}\right)
$$

where the superscripts, in symbolic notation, indicate that the random variables $X_{j}$ and $Y_{j}$ are involved in the conditional probability. For the sake of simplicity, the notations, $y_{j}, x_{j}$, et cetera in the probabilities are used for $Y_{j}=y_{j}, X_{j}=x_{j}$, et cetera.

It is assumed that if the subject has complete knowledge of the answer $\left(x_{j}=1\right)$, the correct alternative is chosen; that is, $\Phi_{1 y_{j}}^{X_{j} Y_{j}}$ must equal 1 if $y_{j}$ is the right alternative and 0 if $y_{j}$ is the wrong alternative. If the subject is in the "Don't know" state $\left(x_{j}=\right.$ $0), \Phi_{0 y_{j}}^{X_{j} Y_{j}}$ can take on any value from 0 to 1 as long as the sum of the probabilities for all values of $y_{j}\left(1\right.$ through $\left.r_{j}\right)$ is 1 .

The latent responses are assumed to be governed by a one-parameter-logistic model (Rasch, 1960), where the probability of latent response $x_{j}$, given that the subject has ability $\theta$, is

$$
P\left(x_{j} \mid \theta\right)=\frac{\exp \left(x_{j}\left(\theta-\delta_{j}\right)\right)}{1+\exp \left(\theta-\delta_{j}\right)},
$$

and $\delta_{j}$ is the difficulty of item $j$. have

Assuming that $y_{j}$ and $x_{j}$ depend on $x_{j}$ and latent ability $\theta$ only, respectively, we

$$
P\left(y_{j} \mid \theta\right)=\frac{\Phi_{0 y_{j}}^{X_{j} Y_{j}}+\Phi_{\Psi_{j}}^{X_{j} Y_{j}} \exp \left(\theta-\delta_{j}\right)}{1+\exp \left(\theta-\delta_{j}\right)} .
$$

In the foregoing, we have indicated that an item exhibits DIF if the probability of a correct response among equally able test takers is different between subgroups. According to (3), this means that if item $j$ exhibits DIF, the attraction parameter $\Phi_{x_{j} y_{j}}^{X_{j} Y_{j}}$ and/or the difficulty parameter $\delta_{j}$ did not have the same value for all subgroups. So the two sources of DIF (attraction of the alternatives and difficulty of the item) are welldefined by the model.

To formulate a complete model, the response pattern of a subject on all the items in a test is denoted by the vector $\mathbf{y}=\left(y_{1}, \ldots, y_{k}\right)$. The vector of latent responses of a subject is denoted by $\mathbf{x}=\left(x_{1}, \ldots, x_{k}\right)$. The corresponding random variables are denoted by $\mathbf{Y}$ and $\mathbf{X}$. Letting $F(\theta)$ be the continuous distribution function of the latent ability $\theta, \delta=\left(\delta_{1}, \ldots, \delta_{k}\right)$ the vector of item difficulties, and $t=x_{1}+\cdots+x_{k}$, the number correct score. Using (1), (2), and the assumption of local independence of the $y_{j}$ and $x_{j}$ variables, the marginal probability of the observed responses $y$ can be written as 


$$
\begin{aligned}
P(\mathbf{y}) & =\sum_{\mathbf{x}} \int_{-\infty}^{\infty} P(\mathbf{y} \mid \mathbf{x}, \theta) P(\mathbf{x} \mid \theta) d F(\theta) \\
& =\sum_{\mathbf{x}}\left(\prod_{j=1}^{k} \Phi_{x_{j} y_{j}}^{X_{j} Y_{j}}\right) \exp \left(-\sum_{j=1}^{k} x_{j} \delta_{j}\right) \int_{-\infty}^{\infty} \exp (t \theta) C(\theta, \delta)^{-1} d F(\theta),
\end{aligned}
$$

where

$$
C(\theta, \delta)=\prod_{j=1}^{k}\left(1+\exp \left(\theta-\delta_{j}\right)\right),
$$

and $\Sigma_{\mathbf{x}}$ means the summation over all possible latent response patterns $\mathbf{x}=\left(x_{1}, \ldots\right.$, $\left.x_{k}\right)$.

To detect DIF in multiple choice items, (4) has to be extended to include subgroups. To keep the main idea of this section in proper perspective, subgroups have been ignored so far, but will be considered in a later section.

In the next section we will formulate the model as an incomplete latent class model. The integral in (4) will then be absorbed into a latent class parameter that depends only on the number correct score $t$, implying that it is not necessary to specify the distribution function $F(\theta)$ any further.

\section{The Model Written as an Incomplete LCA Model}

Kelderman (1988) showed that (4) is an incomplete latent-class model in the sense of Haberman (1979, chap. 10):

$$
P(\mathbf{y})=\sum_{\mathbf{x}} \Phi_{\imath}^{T} \Phi_{x_{1}}^{X_{1}} \cdots \Phi_{x_{k}}^{X_{\ell}} \Phi_{x_{1} y_{1}}^{X_{1} Y_{1}} \cdots \Phi_{x_{k} y_{k}}^{X_{k} Y_{k}}
$$

with

$$
\Phi_{t}^{T}=\int_{-\infty}^{\infty} \exp (t \theta) C(\theta, \mathbf{\delta})^{-1} d F(\theta),
$$

and for $j=1, \ldots, k$,

$$
\Phi_{x_{j}}^{X_{j}}=\exp \left(-x_{j} \delta_{j}\right)
$$

and where the $\Phi$-parameters are subject to the restrictions

$$
\Phi_{x_{j} 1}^{X_{j} Y_{j}}+\cdots+\Phi_{x_{j} r_{j}}^{X_{j} Y_{j}}=1
$$

In this model, each value of $x$ represents a latent class. The model in (5) is incomplete since for certain given values of $\mathbf{X}$, only a limited number of combinations $\left(Y_{1}, \ldots\right.$, $Y_{k}$ ) are possible. Because $\Phi_{t}^{T}$ depends on an underlying latent trait distribution $F(\theta)$, these parameters are subject to the following complex inequality constraints (Cressie \& Holland, 1983; Kelderman, 1984):

$$
\text { det. }\left(\left\|\Phi_{r+s}^{T}\right\|_{r, s=0}^{q_{1}}\right) \geq 0,
$$

and 


$$
\text { det. }\left(\left\|\Phi_{r+s+1}^{T}\right\|_{r, s=0}^{q_{2}}\right) \geq 0
$$

where

$$
\begin{aligned}
& q_{1}= \begin{cases}\frac{k}{2} & \text { if } k \text { is even, } \\
\frac{k-1}{2} & \text { if } k \text { is odd, }\end{cases} \\
& q_{2}= \begin{cases}\frac{k-2}{2} & \text { if } k \text { is even, } \\
\frac{k-1}{2} & \text { if } k \text { is odd }\end{cases}
\end{aligned}
$$

and det. $\left(\|-\|_{r, s=0}^{q}\right)$ defines the determinant of a matrix with row index $r$ and column index $s$, both running from zero to $q$.

Since it is not our goal to fit a model for the data, but only to decide if a certain item exhibits DIF, we will follow Cressie and Holland and ignore these inequality constraints. The resulting model, the so-called generalized Rasch model, provides an easy way to decide whether or not an item exhibits DIF. The generalized Rasch model is also equivalent to the "conditional" Rasch model; that is, a Rasch model in which there is a conditioning on the number correct score (Kelderman, 1984). Incomplete table methodology can be used to formulate several hypotheses about DIF by specifying alternative models that contain additional subgroup-dependent parameters.

\section{Testing for DIF using Related LCA Models}

An item can show DIF in two different ways. First, as indicated before, an item exhibits DIF if equally able individuals from different subgroups have different probabilities of "Knowing" the answer. This will be referred to as DIF in the latent response. It was assumed earlier that if subjects are in the "Know" state, they will choose the correct alternative. But if subjects are in the "Don't know" state, they may choose any of the alternatives. Therefore, an item also exhibits DIF if the attractiveness of the alternatives varies from subgroup to subgroup conditioned on ability. This will be referred to as DIF in the attraction parameters.

To detect DIF, the model in (5) is reformulated as

$$
P(\mathbf{y} \mid i)=\sum_{\mathbf{x}} \Phi_{i t}^{I T} \Phi_{i x_{1}}^{I X_{1}} \cdots \Phi_{i x_{k}}^{I X_{k}} \Phi_{i x_{1} y_{i}}^{I X_{1} Y_{1}} \cdots \Phi_{i x_{k} y_{k}}^{I X_{k} Y_{k}},
$$

where $P(y \mid i)$ is the conditional distribution of observed response $\mathbf{y}$ given observed subgroup $i(i=1, \ldots, g)$ and each term on the right side is equal to the corresponding term on the right side of (5), extended with the subgroup. For example,

$$
\Phi_{i t}^{I T}=\int_{-\infty}^{\infty} \exp (t \theta) C\left(\theta, \boldsymbol{\delta}_{i}\right)^{-1} d F_{i}(\theta),
$$

where 


$$
C\left(\theta, \boldsymbol{\delta}_{i}\right)=\prod_{j=1}^{k}\left(1+\exp \left(\theta-\delta_{i j}\right)\right),
$$

$\delta_{i j}$ is the difficulty of item $j$ in group $i$, and $F_{i}(\theta)$ is the continuous distribution function of the latent ability $(\theta)$ in group $i$.

In the model in (7), all items are considered to exhibit DIF both in the latent response and the attraction parameters. If some items exhibit DIF neither in the latent response nor in the attraction parameters, then the $\Phi$-parameters for these items are restricted. For example, if in a certain model, Item 1 exhibits no DIF in the latent response, then the $\Phi_{i x_{1}}^{I X_{1}}$ parameters are restricted in the following manner:

$$
\Phi_{1 x_{1}}^{I X_{1}}=\cdots=\Phi_{g x_{1}}^{I X X_{1}} .
$$

In the next subsections, models are formulated for studying the two types of DIF.

\section{DIF in the Latent Response}

To test whether the interaction between subgroup $i$ and the latent response to Item 1 is zero (i.e., whether Item 1 exhibits DIF in the latent response), an alternative model is formulated as

$$
P(\mathbf{y} \mid i)=\sum_{\mathbf{x}} \Phi_{i t}^{I T} \Phi_{i x_{1}}^{I X_{1}} \Phi_{x_{2}}^{X_{2}} \cdots \Phi_{x_{k}}^{X_{k}} \Phi_{x_{1} y_{1}}^{X_{1} Y_{1}} \cdots \Phi_{x_{k} y_{k}}^{X_{k} Y_{k}}
$$

The model in (8) can be obtained from (7) by setting all $\Phi$-parameters, excluding the difficulty parameter of Item 1, equal for all subgroups:

$$
\Phi_{1 x_{j}}^{I X_{j}}=\cdots=\Phi_{g x_{j}}^{I X_{j}}=\Phi_{x_{j}}^{X_{j}}, \quad(j=2, \ldots, k)
$$

and

$$
\Phi_{1 x_{j} y_{j}}^{I X_{j} Y_{j}}=\cdots=\Phi_{g x_{j} y_{j}}^{I X_{j} Y_{j}}=\Phi_{x_{j} y_{j}}^{X_{j} Y_{j}} . \quad(j=1, \ldots, k)
$$

This alternative model is compared with

$$
P(\mathbf{y} \mid i)=\sum_{\mathbf{x}} \Phi_{i t}^{I T} \Phi_{x_{1}}^{X_{1}} \cdots \Phi_{x_{k}}^{X_{k}} \Phi_{x_{1} y_{1}}^{X_{1} Y_{1}} \cdots \Phi_{x_{k} y_{k}}^{X_{k} Y_{k}},
$$

where all $\Phi$-parameters are set equal across subgroups. If a statistical test of the difference between the models is significant, it may be concluded that the difficulty of Item 1 varies from subgroup to subgroup. In this case, subjects in one subgroup may find it more difficult to solve the problem than subjects in another subgroup.

\section{DIF in the Attraction Parameters}

To test the null hypothesis that the interaction between the subgroup and the observed response to Item 1 is zero (i.e., whether Item 1 exhibits DIF in the attraction parameters), (9) is compared with the alternative model

$$
P(\mathbf{y} \mid i)=\sum_{\mathbf{x}} \Phi_{i t}^{I T} \Phi_{x_{1}}^{X_{1}} \cdots \Phi_{x_{k}}^{X_{k}} \Phi_{i x_{1} y_{1}}^{I X_{1} Y_{1}} \Phi_{x_{2} y_{2}}^{X_{2} Y_{2}} \cdots \Phi_{x_{k} y_{k}}^{X_{k} Y_{k}}
$$

where, similar to (8), all $\Phi$-parameters, except for the attraction parameters for Item 1, are set equal across subgroups. If the statistical test is significant, it may be concluded that the attractiveness of the Item 1 alternatives varies from subgroup to subgroup. In 
(8) and (10), the $\Phi$-parameters are specified to test for DIF for only one item. Obviously, similar model terms can be specified for two or more items if necessary. It is also possible to define models in which one item exhibits DIF in the latent response and another (or the same) item exhibits DIF in the attraction parameters.

\section{Parameter Estimation and Model Testing}

Let $n_{i \mathbf{x y}}$ be the number of individuals in subgroup $i$ with $\mathbf{X}=\mathbf{x}$ and $\mathbf{Y}=\mathbf{y}$ under a certain model and let $m_{i \mathbf{x y}}=N P(i, \mathbf{x}, \mathbf{y})$ be the expected value of $n_{i \mathbf{x y}}$. Although $n_{i \mathbf{x y}}$ is not observed, it is possible to estimate the means $m_{i \mathbf{x y}}$ of $n_{i \mathbf{x y}}$, and the $\Phi$-parameters from the observed $n_{i y}$ (or $n_{\mathrm{y}}$ if the subgroup is unobserved) by the method of maximum likelihood. To illustrate this, consider the model in (7). The maximum-likelihood equations for (7) would be (Haberman, 1979):

$$
\hat{m}_{i t}^{I T}=\hat{n}_{i t}^{I T}, \quad \hat{m}_{i x_{j} y_{j}}^{I X_{j} Y_{j}}=\hat{n}_{i x_{j} y_{j}}^{I X_{j} Y_{j}}, \quad(j=1, \ldots, k),
$$

where

$$
\hat{n}_{i \mathbf{x y}}=\left(\frac{\hat{m}_{i \mathbf{x y}}}{\hat{m}_{i \mathbf{y}}^{I \mathbf{Y}}}\right) n_{i \mathbf{y}}^{I \mathbf{Y}},
$$

and $n_{i t}^{I T}$ and $n_{i x_{j} y_{j}}^{I X_{j} Y_{j}}$ are the numbers of individuals in subgroup $i$ with $T=t, X_{j}=x_{j}$, and $Y_{j}=y_{j}$, respectively. Furthermore, $m_{i t}^{I T}$ and $m_{i x_{j} y_{j}}^{I X_{j} Y_{j}}$ are the expected values of $n_{i t}^{I T}$ and $n_{i x_{j} y_{j}}^{I X_{j} Y_{j}}$, respectively. If the subgroup $i$ is not observed, then $n_{i y}$ and $m_{i y}$ in (11) have to be replaced by $n_{y}$ and $m_{y}$, respectively. The maximum-likelihood equations can be solved by the iterative proportional fitting algorithm or the scoring algorithm (Goodman, 1978; Haberman, 1979). The iterative proportional fitting algorithm is preferred, because it is less sensitive to the choice of starting values. Similar estimation equations can be formulated for the restricted models.

The overall goodness-of-fit of an incomplete latent-class model can be tested by the Pearson statistic $(Q)$ or the likelihood-ratio statistic (LR; see Haberman, 1979). Both statistics are asymptotically distributed as chi-square with degrees of freedom equal to the difference between the count $n_{\mathrm{y}}$ (or $n_{i y}$ if the subgroup is observed) and the number of estimable parameters. The number of estimable parameters of a model should be equal to the rank of the information matrix (Goodman, 1978; McHugh, 1956).

By the difference in the likelihood-ratio test statistics for two models (LR(a;b)), it can be tested whether the alternative model $b$ yields a significant improvement in fit over the restricted model $a$, which is a special case of model $b$. Under the assumption of model $a, \operatorname{LR}(\mathrm{a} ; \mathrm{b})$ is asymptotically chi-square distributed with degrees of freedom equal to the difference in the numbers of estimable parameters of both models (Bishop, Fienberg, \& Holland, 1975).

\section{An Empirical Example}

As an example, four items from the Second International Mathematics Study in the Netherlands were analyzed (Eggen, Pelgrum, \& Plomp, 1987). Each item was a fivechoice item with only one correct alternative. A sample of 3002 students was drawn from two types of schools for lower secondary education in the Netherlands. To illustrate the use of quasi-loglinear models for detecting DIF, the students' level of education was chosen as the grouping variable: subgroup MAVO (intermediate general ed- 
TABLE 1

Likelihood-Ratio Tests for Detecting DIF in the Data from the

Second International Mathematics Study

\begin{tabular}{|c|c|c|c|c|}
\hline \multirow[b]{2}{*}{ Item } & \multicolumn{2}{|l|}{$\begin{array}{c}\text { DIF in the } \\
\text { Latent Response }\end{array}$} & \multicolumn{2}{|c|}{$\begin{array}{c}\text { DIF in the } \\
\text { Attraction Parameters }\end{array}$} \\
\hline & Likelihood-Ratio & DF & Likelihood-Ratio & DF \\
\hline 1 & 1.701 & 1 & $26.519^{*}$ & 4 \\
\hline 2 & $4.720^{*}$ & 1 & $21.340^{*}$ & 4 \\
\hline 3 & 1.747 & 1 & 6.033 & 4 \\
\hline 4 & .018 & 1 & $52.595^{*}$ & 4 \\
\hline
\end{tabular}

Note: Tests marked with an asterisk are significant $(\alpha=.05)$.

ucation) and subgroup HAVO/VWO (higher general education and pre-university education).

The models in (8) and (10) were fitted to the data using the computer-program LCAG (Hagenaars \& Luijkx, 1987). LCAG is a program for estimating the parameters of loglinear models with latent variables, and yields, besides the estimated latent conditional probabilities (i.e., the attraction parameters), the estimated expected frequency distribution of the latent variables within the model. From this frequency distribution the difficulty parameters were estimated using LOGIMO (Kelderman \& Steen, 1988). LOGIMO is a general computer program especially written for analyzing loglinear IRT models. Both programs use the efficient IPF algorithm for estimating the parameters.

In the first series of analyses, each item was separately tested for DIF in the latent response or in the attraction parameters. For example, to test if Item 1 exhibited DIF in the latent response, we postulated that the difficulty parameter of Item 1 was the only parameter that varied over the two groups. The models in (8) and (10) were compared to (9) to test for DIF in the latent response and for DIF in the attraction parameters, respectively. Table 1 shows the values of the likelihood-ratio test and the degrees of freedom for the models in (8) and (10), separately for each item. For both tests, group membership (i.e., the level of education) was treated as known.

From Table 1 it may be concluded that, except for Item 2, the item difficulty parameters do not vary significantly between the two subgroups (MAVO and HAVO/ 
TABLE 2

Attraction Parameters for the Alternatives of the Four Items

\begin{tabular}{|c|c|c|c|c|c|}
\hline & \multicolumn{5}{|c|}{ Alternatives } \\
\hline Item & A & $\mathbf{B}$ & $\mathbf{C}$ & $\mathbf{D}$ & $\mathbf{E}$ \\
\hline & \multicolumn{5}{|c|}{ Subgroup HAVO/VWO } \\
\hline 1 & .073 & .033 & .685 & .174 & .035 \\
\hline 2 & .743 & .123 & .061 & .045 & .028 \\
\hline 3 & .106 & .296 & .146 & .367 & .085 \\
\hline \multirow[t]{2}{*}{4} & .110 & .355 & .235 & .092 & .208 \\
\hline & \multicolumn{5}{|c|}{ Subgroup MAVO } \\
\hline 1 & .211 & .024 & .563 & .193 & .009 \\
\hline 2 & .662 & .240 & .068 & .015 & .015 \\
\hline 3 & .140 & .468 & .122 & .111 & .159 \\
\hline 4 & .068 & .241 & .341 & .084 & .266 \\
\hline
\end{tabular}

Note: The correct alternatives are underlined.

VWO). Only Item 2 exhibits DIF in the latent response. When we take a closer look at the difficulty of Item 2 , we can be see that it was substantially smaller for MAVOstudents $\left(\delta_{22}=1.90\right)$ than for HAVO/VWO-students $\left(\delta_{12}=0.82\right)$. The difficulty parameters of the other three Items 1,3 , and 4 were $-1.52,-3.54$, and 1.32 , respectively. Note that these items exhibited no DIF in the latent response; therefore, the difficulty parameters were estimated by setting the item parameters equal in both subgroups.

The likelihood-ratio tests reported in Table 1 also indicates that the attractiveness of the alternatives to Items 1, 2, and 4 were significantly different in both subgroups. Estimates of the attraction parameters for the alternatives of each item are presented in Table 2. These results indicate that a HAVO/VWO-student is more likely to choose the correct alternative to Item 1 than a MAVO-student. On the other hand, a MAVO- 
student is more likely to choose the correct alternative for Item 2 , because the associated attraction parameter of the correct alternative for Item 2 in this group is twice as big as the associated attraction parameter for a HAVO/VWO-student. For both subgroups, however, the correct alternative is not the most attractive alternative.

The attraction parameters for the correct alternative of Item 4 are approximately the same for both subgroups, but for the alternatives B and C, there is a curious difference between the two subgroups. A HAVO/VWO-student would guess alternative $B$ with almost the same probability as a MAVO-student would guess alternative $C$, and would guess alternative $C$ with almost the same probability as a MAVO-student would guess alternative B. Item 3 exhibits no DIF in the attraction parameters. However, the attraction parameter for the right alternative in the subgroup HAVO/VWO is more than three times as big as the associated attraction parameter in the subgroup MAVO. Nevertheless, this difference had no significant effect on the test for DIF in the attraction parameters, because the item was very easy for both subgroups.

A major problem in DIF studies is the explanation of DIF. Although it is beyond the scope of this paper, a tentative explanation for the observed DIF in the attraction parameters of Item 4 is the familiarity with the mathematical terms. In Item 4 (see Appendix 1) the subject has to give the definition of a parallelogram. Since the attraction parameters for the alternative $A, D$, and $E$ are approximately the same for the two subgroups (see Table 2), the observed DIF in the attraction parameters is probably caused by the alternatives B and C. Knowing the formulation of Item 4 we can conclude that a MAVO student is probably more familiar with the mathematical terms axis of symmetry and diagonal than a HAVO/VWO student.

In the foregoing analyses, the two types of DIF were studied separately. Also, only one item was evaluated at a time. As indicated earlier, it is possible to analyze models in which more than one item exhibits DIF. To illustrate this possibility, a model in which Items 1, 2, and 4 exhibit DIF in the attraction parameters and Item 2 exhibits DIF in the latent response, was considered. This model shows considerable improvement in fit, compared to the model in (9) (likelihood-ratio is 100.5 with 13 degrees of freedom). From Table 1 it also follows that this model fits the data better than the models discussed previously. The estimated parameters, however, do not differ much from the estimated parameters of the previous models; therefore, they are not given.

In summary, the difficulty of the four items can be ordered in the following way: $\delta_{3}<\delta_{1}<\delta_{4}<\delta_{2}$. That is, Item 3 is the easiest and Item 2 is the most difficult. The attractiveness of alternatives of Items 1,2 , and 4 as well as the difficulty of Item 2 are not the same for the two subgroups. Item 3 exhibits no DIF in the latent response or in the attraction parameters.

\section{Discussion}

In this paper an incomplete latent class model is proposed to examine DIF in multiple-choice items through a combination of the usual notion of DIF for correct/ incorrect responses and information about DIF contained in each of the alternatives. In the proposed method a distinction is made between a "Know" state in which the subject has complete knowledge of the answer and a "Don't know" state. It is assumed that if the subject is in the "Know" state, the subject will give the correct answer. The probability that the subject is in the "Know" state is assumed to be governed by the Rasch model. And, if the subject is in the "Don't know" state, the subject will choose the most attractive alternative, where the attractiveness of the alternatives may be different for different alternatives, including the correct one. The model is extended with variables defining subgroups to study DIF. One of the main advantages of the 
proposed method is that it is not only possible to test if a certain item exhibits DIF, but it is also possible to test whether this DIF is caused by the difficulty of the item, the attraction of the alternatives, or both.

In most applications, the subgroup membership is determined by an observed variable (e.g., sex). In some situations, however, subgrouping is suspected but the variable determining subgroup membership is not observable (Kelderman \& Macready, 1990; Mislevy \& Verhelst, 1990). When subgroup membership is unobserved, the subgroup variable in the proposed method is also treated as a latent variable.

In this paper all tests of DIF are two-sided. This means that it is not possible to test directional hypotheses about DIF. The estimated difficulty parameters and the estimated attraction parameters provide only an indication of the direction of DIF. However, together with the knowledge of the item, these estimated parameters may provide the test-constructor a better feel for why an item exhibits DIF or does not exhibit DIF. Further, if many items in a test show DIF, it might be that DIF in one of the items in favor of a subgroup is compensated by DIF in another item in favor of another subgroup. Although DIF in the attraction parameters may have no effect on test scores, it might indicate that the item was functioning differently for the different subgroups.

At the present time the proposed method is not very practical for a large number of polytomous items. This problem is due to the computer program LCAG, which uses in our case such a large amount of memory-space that it is impossible to consider more than four five-choice items at a time. A line of future research should be to develop an estimation method which can handle many items. Future research should also address the question of whether a given model is identified.

\section{Appendix 1}

The English version of Item 4

A quadrilateral MUST be a parallelogram if it has
A. one pair of adjacent sides equal
B. one pair of parallel sides
C. a diagonal as axis of symmetry
D. two adjacent angles equal
E. two pairs of parallel sides

\section{References}

Baker, F. B. (1977). Advances in item analysis. Review of Educational Research, 47, 151-178.

Berk, R. A. (1982). Handbook of methods for detecting test bias. Baltimore: The Johns Hopkins University Press.

Binet, A., \& Simon, T. (1916). The development of intelligence in children. Baltimore: Williams \& Wilkins.

Bishop, Y. M. M., Fienberg, S. E., \& Holland, P. W. (1975). Discrete multivariate analysis. Cambridge, MA: MIT Press.

Bock, R. D. (1972), Estimating item parameters and latent proficiency when the responses are scored in two or more nominal categories. Psychometrika, 37, 29-51.

Clogg, C. C. (1981). Latent structure models of mobility, American Journal of Sociology, 86, 836-868.

Cressie, N., \& Holland, P. W. (1983). Characterising the manifest probabilities of latent trait models. Psychometrika, 48, 129-142.

Eggen, T. J. H. M., Pelgrum, W. J., \& Plomp, Tj. (1987). The implemented and attained mathematics curriculum: Some results of the second international mathematics study in the Netherlands. Studies in Educational Evaluation, 13, 119-135.

Goodman, L. A. (1978). Analyzing qualitative/categorical data: Loglinear models and latent structure analysis. London: Addison Wesley.

Green, B. F., Crone, C. R., \& Folk, V. G. (1989). A method for studying differential distractor functioning. Journal of Educational Measurement, 26, 147-160. 
Haberman, S. J. (1979). Analysis of qualitative data: New developments, Vol. 2. New York: Academic Press.

Hagenaars, J., \& Luijkx, R. (1987). LCAG: latent-class models and other loglinear models with latent variables (Working Paper 17). Tilburg: Tilburg University.

Holland, P. W., \& Thayer, D. (1986). Differential item performance and the Mantel-Haenszel statistic. Paper presented at the Annual Meeting of the American Educational Research Association, San Francisco.

Kelderman, H. (1984). Loglinear Rasch model tests. Psychometrika, 49, 223-245.

Kelderman, H. (1988). An IRT model for item responses that are subject to omission andlor intrusion errors (Research Report 88-16). Enschede: University of Twente.

Kelderman, H. (1989). Item bias detection using loglinear IRT. Psychometrika, 54, 681-697.

Kelderman, H., \& Macready, G, B. (1990). The use of loglinear models for assessing differential item functioning across manifest and latent examinee groups. Journal of Educational Measurement, 27, 307-327.

Kelderman, H., \& Steen, R. (1988), LOGIMO 1: Loglinear item response theory modeling. Computer manual, University of Twente, Department of Educational Technology.

Lazarsfeld, P. F., \& Henry, N. W. (1968). Latent structure analysis, Boston: Houghton-Miffin.

Lord, F. M. (1980). Applications of item response theory to practical testing problems. Hillsdale, $\mathrm{NJ}$ : Lawrence Erlbaum.

McHugh, R. B. (1956). Efficient estimation and local identification in latent-class analysis. Psychometrika, $21,331-347$.

Mellenbergh, G. J. (1982). Contingency table methods for assessing item bias. Journal of Educational Statistics, $7,105-118$.

Mislevy, R. J., \& Verhelst, N. (1990). Modeling item responses when different subjects employ different solutions strategies. Psychometrika, 55, 195-216.

Muthén, B., \& Lehman, J. (1985). Multiple group IRT modeling: Applications to item bias analysis. Journal of Educational Statistics, 10, 133-142.

Osterlind, S. J. (1983). Test item bias. Beverly Hills: Sage.

Rasch, G. (1960). Probabilistic models for some intelligence and attainment tests. Chicago: The University of Chicago Press.

Rudner, L. M., Getson, P. R., \& Knight, D. L. (1980). Biased item detection techniques. Journal of Educational Statistics, 5, 213-233.

Scheuneman, J. (1979). A method of assessing bias in test items. Journal of Educational Measurement, 16, 143-152.

Thissen, D., Steinberg, L., \& Fitzpatrick, A. R. (1989). Multiple choice models: The distractors are also part of the item. Journal of Educational Measurement, 26, 161-176.

Thissen, D., Steinberg, L., \& Wainer, H. (in press). Detection of differential item functioning using the parameters of item response models. In P. W. Holland \& H. Wainer (Eds.), Differential item functioning: Theory and practice. Hillsdale, NJ: Lawrence Erlbaum Associates.

Veale, J. R., \& Foreman, D. I. (1983). Assessing cultural bias using foil response data: cultural variation. Journal of Educational Measurement, 20, 249-258.

Wright, B. D., Mead, R. J., \& Draba, R. (1975). Detecting and correcting test item bias with a logistic response model (RM 22). Chicago: University of Chicago, Department of Education, Statistical Laboratory.

Manuscript received $3 / 1 / 90$

Final version received $4 / 8 / 91$ 